\title{
SYSTEMIC SCLEROSISPATIENTS' ORAL HEALTH: A PATIENT EDUCATION BOOKLET REPORT
}

Thalles Guilherme de Almeida ${ }^{1}$ Caio Carvalhais Chaves $^{1}$, Barbara Niquini Assunção², João Victor de Pinho Costa ${ }^{1}$, Vivian Guerra de Faria ${ }^{1}$, Luiza Castro Fernandes ${ }^{1}$, Alessandra Rodrigues Hansen Ferreira ${ }^{1}$, Anderson Ramos Lisboa ${ }^{1}$, Felipe Souza da Silva ${ }^{1}$, Liliane Amaral ${ }^{1}$, Maria Raquel Costa Pinto ${ }^{1}$, Leandro Augusto Tanures ${ }^{1}$, Flávia Patrícia Sena Teixeira Santos ${ }^{1}$, Gilda Aparecida Ferreira ${ }^{1}$, Junia Amorim Andrade ${ }^{1}$, Débora Cerqueira Calderaro ${ }^{1, *}$

1.Universidade Federal de Minas Gerais, Belo Horizonte (MG), Brazil; 2. Faculdade de Ciências Médicas de Minas Gerais, Belo Horizonte (MG), Brazil.

*Corresponding author: dccalderaro@gmail.com

\section{BACKGROUND}

Systemic sclerosis (SSc) is a rare autoimmune disease characterized mainly by excessive collagen deposition in the skin and multiple internal organs. The orofacial region is affected in $80 \%$ of SSc cases and may lead to microstomia, xerostomia, periodontal diseases, caries and edentulism. The production of patient education booklets helps to spread information on measures to prevent these complications and maintain functionality, as well as alerts to the need for regular follow-up with qualified professionals.

\section{METHODS}

In the evaluation of a previous booklet, a demand for information regarding oral health in SSc was identified. Then, a multidisciplinary team researched the scientific literature and developed a booklet with explanations on orofacial changes in SSc and suggestions on exercises to improve oral mobility, measures and adaptations for good oral hygiene, guidelines for adequate cleaning of dental prostheses and alternatives for the relief of xerostomia. The booklet was submitted to evaluation for people with SSc, through a digital form on the Google Forms platform, which questioned the understanding of the text and illustrations, requested a grade from 0 to 10 for the booklet in terms of relevance, explicitness and aesthetics, and asked for suggestions for improving the material.

\section{RESULTS}

The booklet was evaluated by 120 SSc people. One hundred and one (84.7\%) patients stated the material presented new relevant information. There were no difficulties in understanding the text and illustrations. The booklet received an average score of 9.73 (SD \pm 0.80 ) in relevance, 9.82 ( $S D \pm 0.47$ ) in explicitness and $9.49(S D \pm 0.93)$ in aesthetics. The final version (Figures $1-8)$ was emailed to the participants, posted on social networks, and published on the university's website.

\section{CONCLUSION}

The development of educational materials on oral health for patients with SSc is relevant, as it supports the prevention of dental diseases, avoiding the need for dental procedures. Guidance on exercises for oral mobility can help to prevent loss of functionality for speech, nutrition and oral hygiene. During the social distancing imposed by the COVID-19 pandemic, the online spread of the booklet is valuable since it allows reaching a greater number of people. Given the good evaluation of the booklet by the patients, we believe that the objectives of this educational material were achieved.

\section{KEYWORDS}

Systemic sclerosis, Patient education as topic, Oral health, Care, Self. 


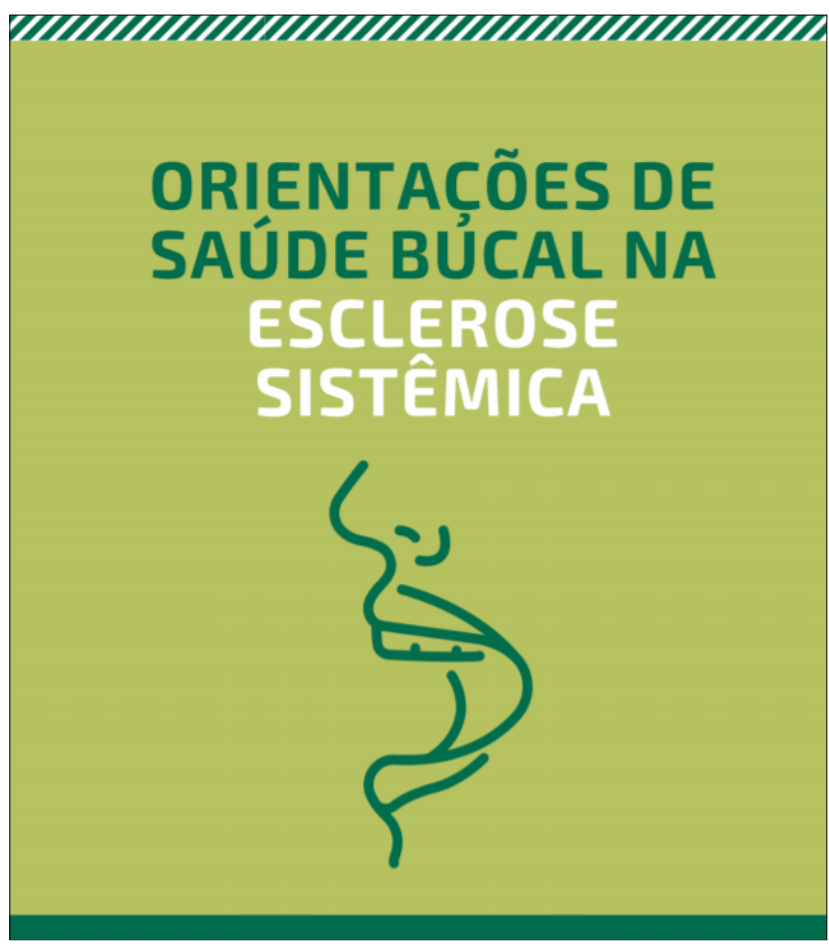

Figure 1. Cover of the booklet.

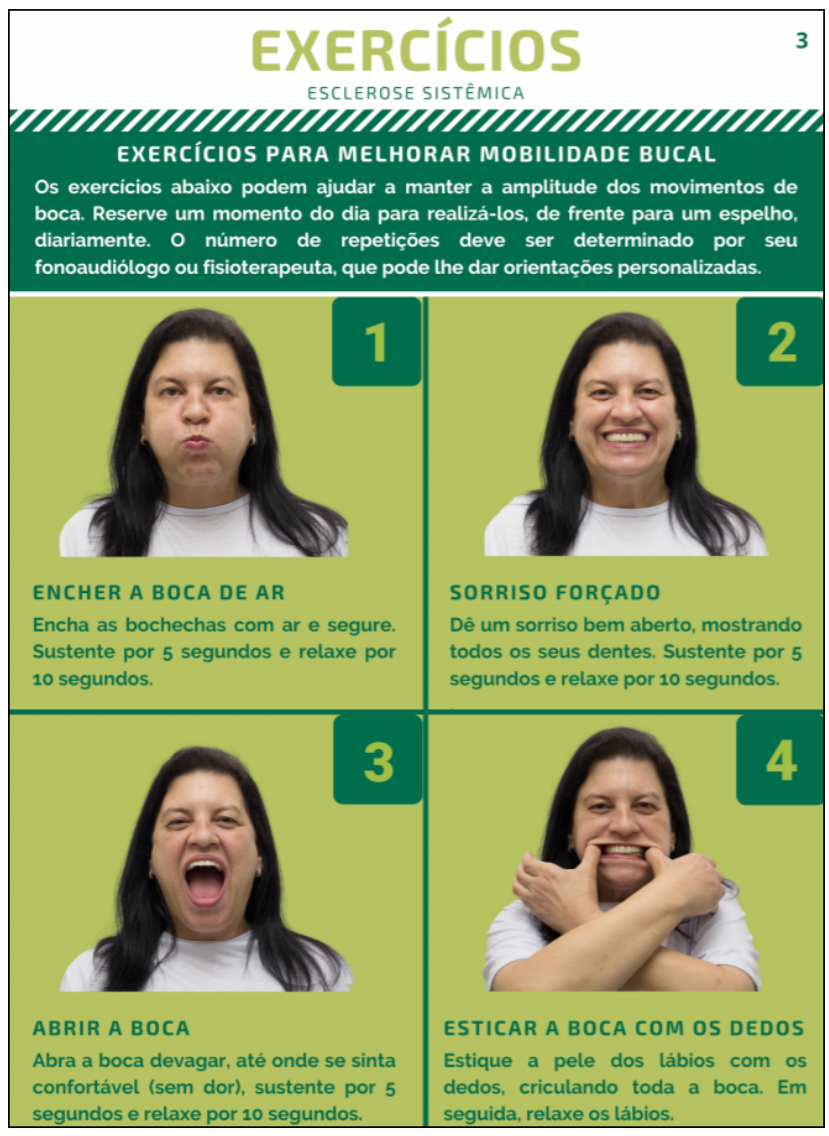

Figure 3. Page 3 of the booklet. Suggestions of exercises to improve oral mobility.

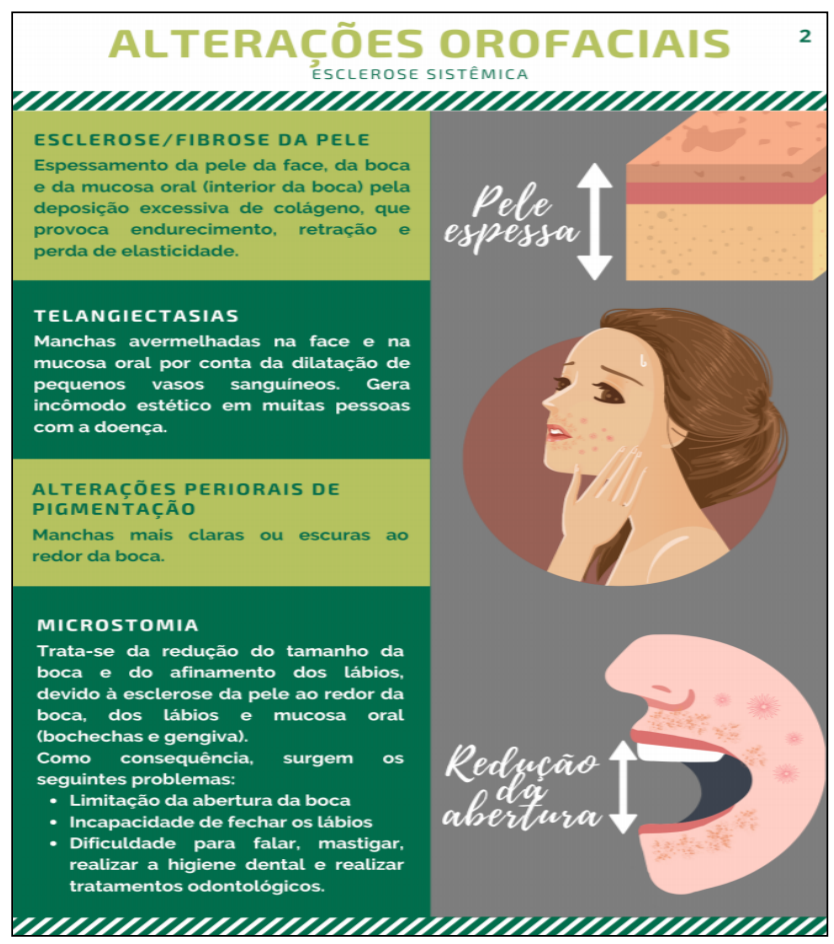

Figure2.Page2 of the booklet.Description of themain orofacial changesinSSc.

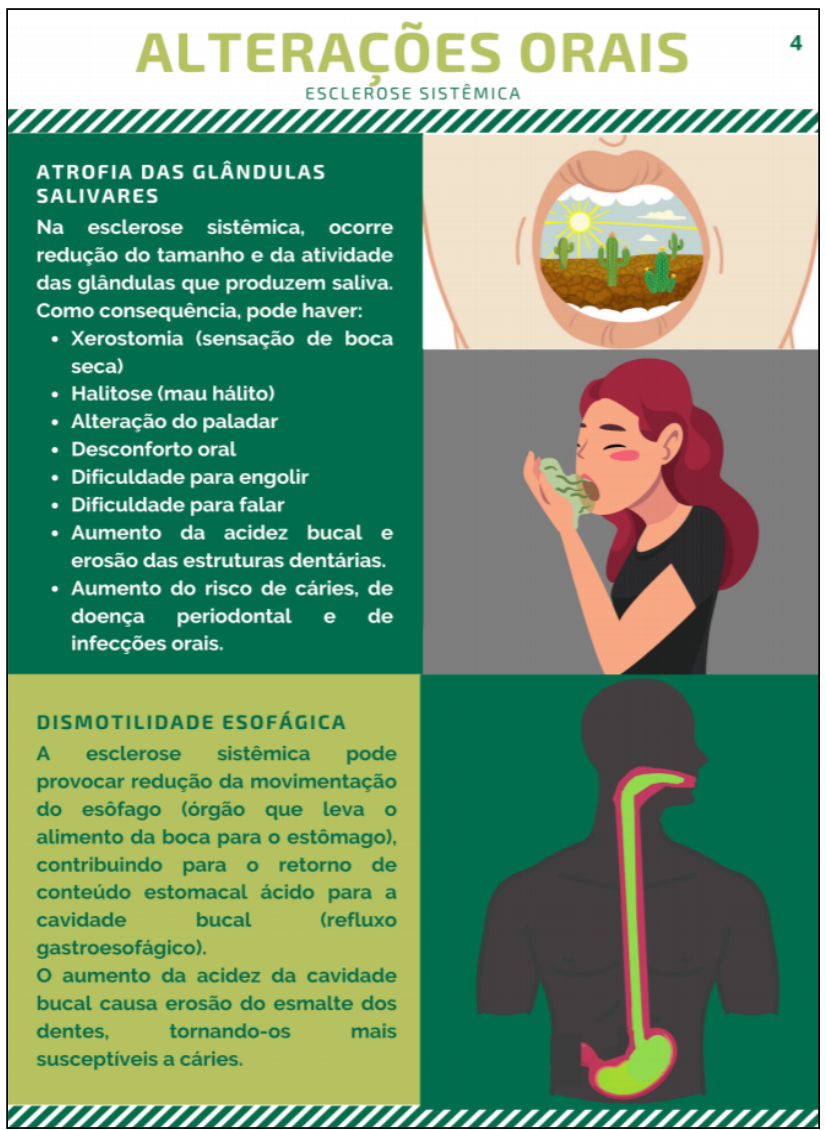

Figure 4. Page 4 of the booklet. Explanations of salivary glands hypofunction and esophageal dysmotility in SSc. 


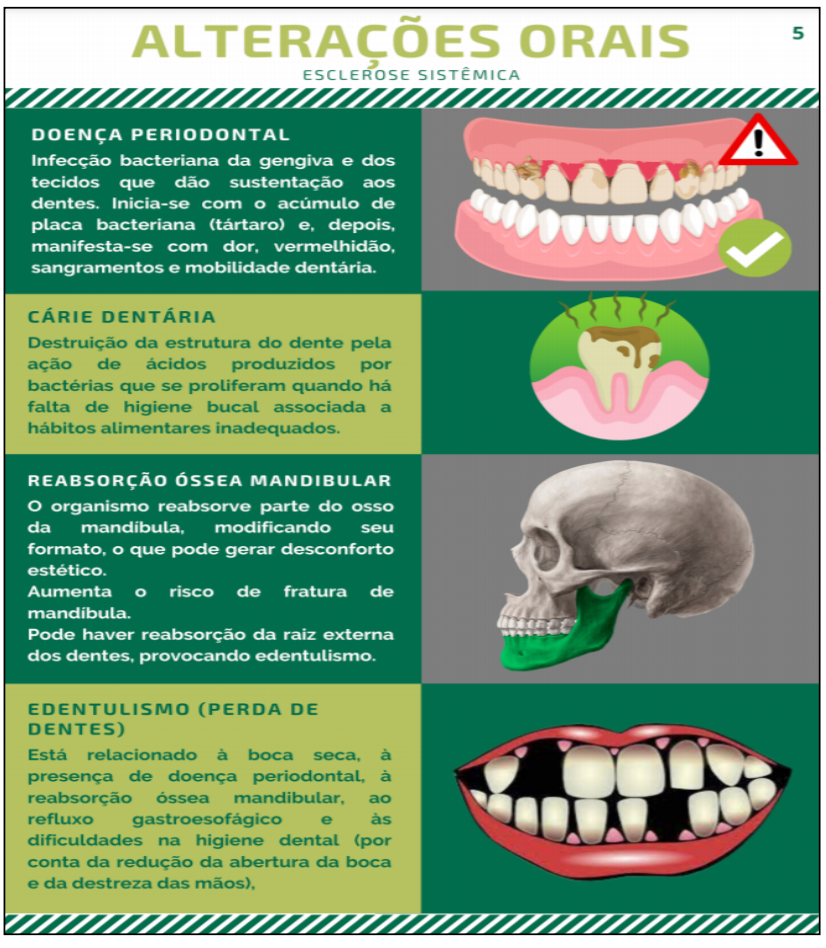

Figure 5. Page 5 of the booklet. Description of dental diseases, mandibular bone resorption and edentulism in SSc.

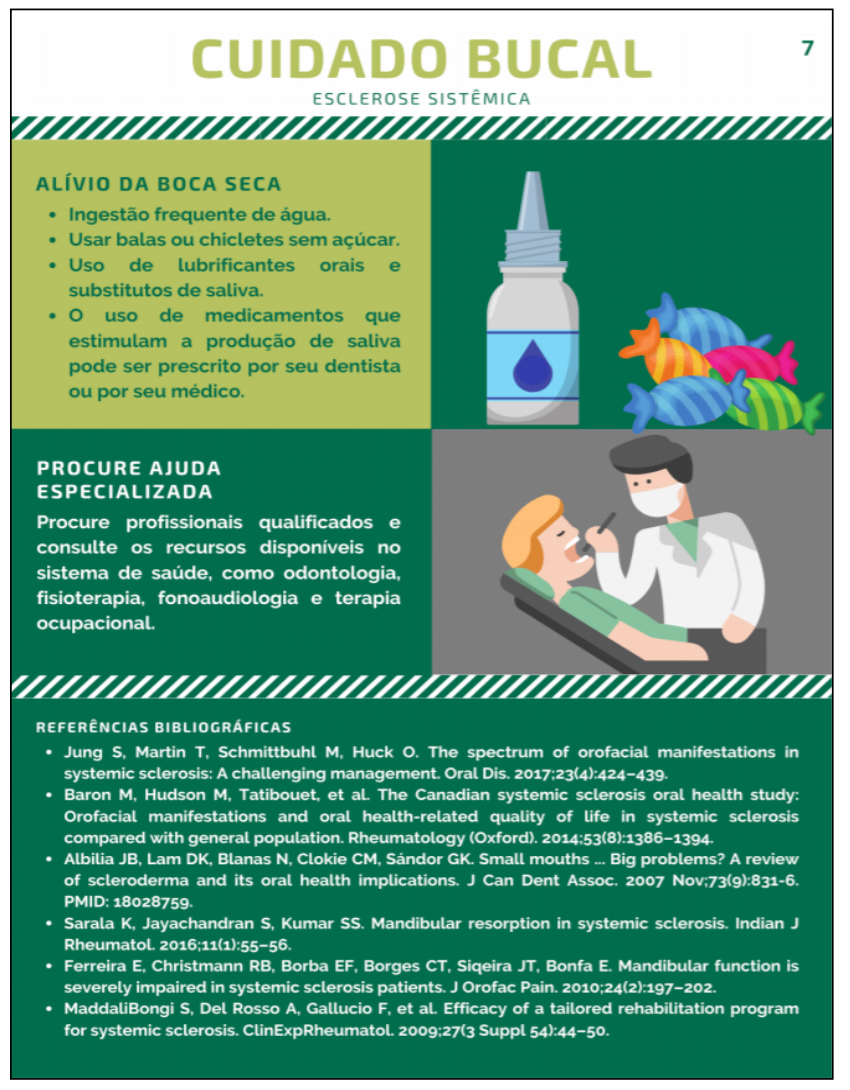

Figure 7. Page 7 of the booklet. Description of measures for xerostomia relief and alerts to the need for regular follow-up with qualified professionals.

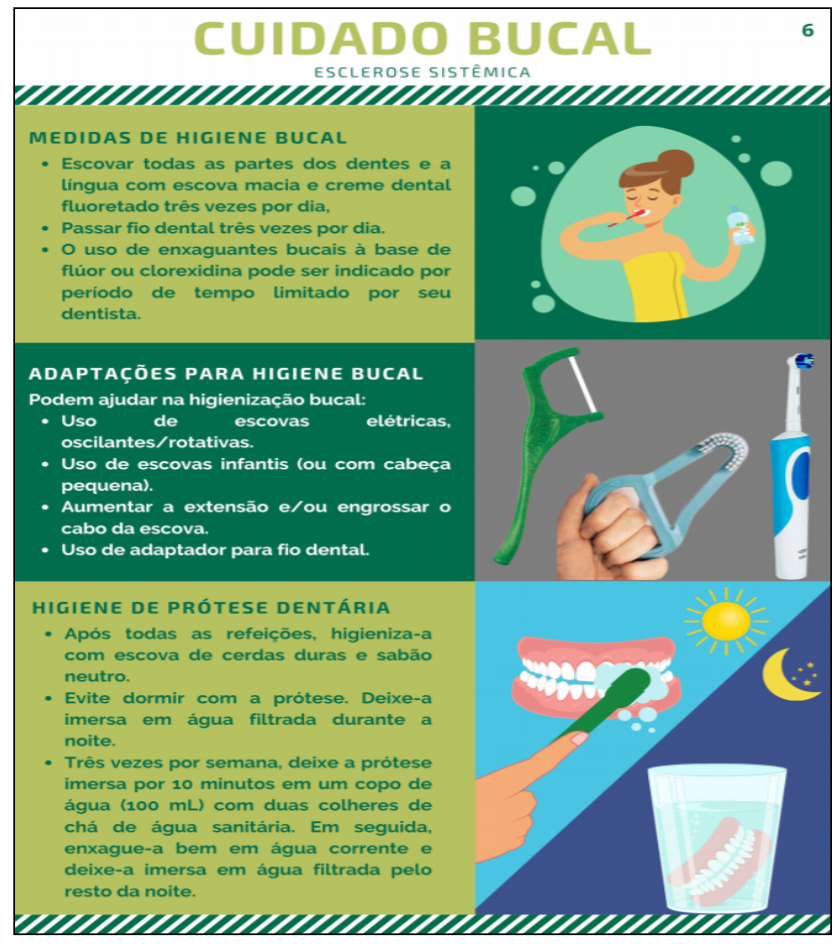

Figure 6. Page 6 of the booklet. Suggestions of measures and adaptations for good oral hygiene and adequate handling and cleaning of dental prostheses.

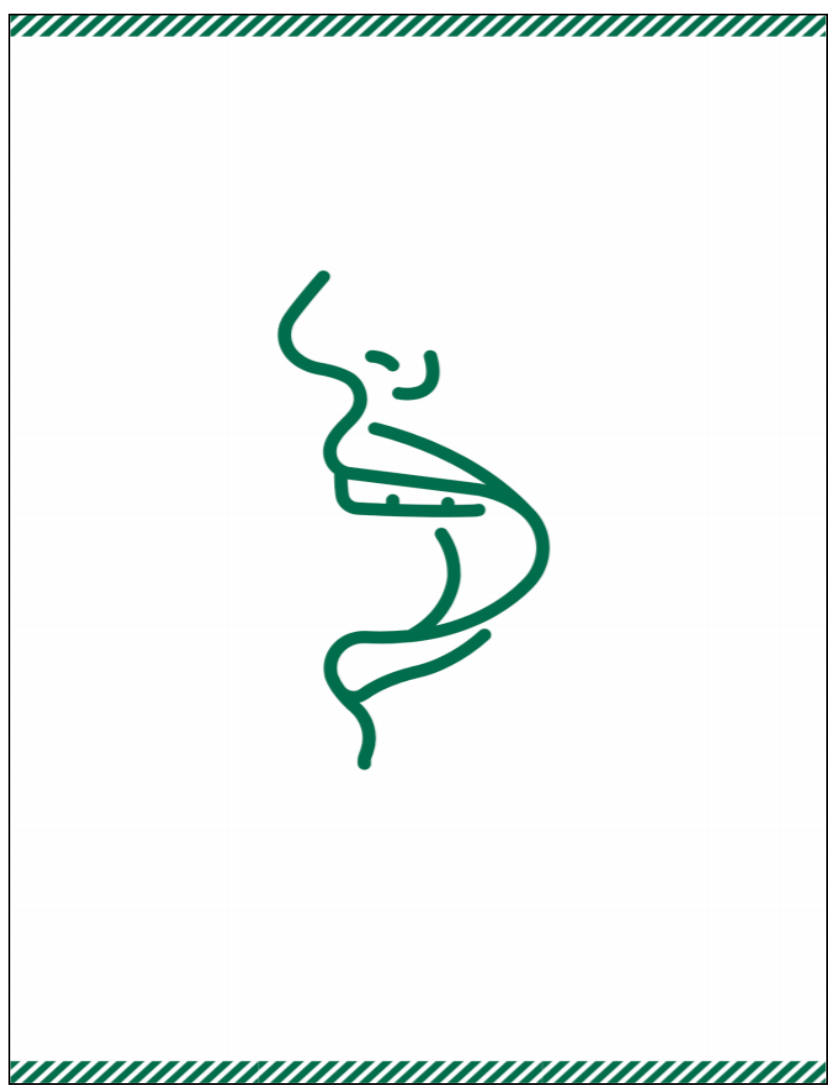

Figure 8. Back cover of the booklet. 